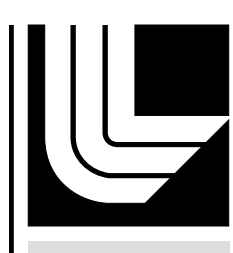

LAWRENCE LIVERM ORE N A TIO NAL LABORATORY

\title{
The National Ignition Campaign: Status and Progress
}

E. Moses

August 21, 2012

24th International IAEA Fusion Energy Conference San Diego, CA, United States October 8, 2012 through October 13, 2012 
This document was prepared as an account of work sponsored by an agency of the United States government. Neither the United States government nor Lawrence Livermore National Security, LLC, nor any of their employees makes any warranty, expressed or implied, or assumes any legal liability or responsibility for the accuracy, completeness, or usefulness of any information, apparatus, product, or process disclosed, or represents that its use would not infringe privately owned rights. Reference herein to any specific commercial product, process, or service by trade name, trademark, manufacturer, or otherwise does not necessarily constitute or imply its endorsement, recommendation, or favoring by the United States government or Lawrence Livermore National Security, LLC. The views and opinions of authors expressed herein do not necessarily state or reflect those of the United States government or Lawrence Livermore National Security, LLC, and shall not be used for advertising or product endorsement purposes. 


\title{
The National Ignition Campaign: Status and Progress
}

\author{
E. I. Moses \\ Lawrence Livermore National Laboratory, Livermore, CA 94450
}

\begin{abstract}
The National Ignition Facility (NIF) at Lawrence Livermore National Laboratory (LLNL) has been operational since March 2009 and a variety of experiments have been completed and many more are planned in support of NIF's mission areas: national security, fundamental science, and fusion energy. NIF capabilities and infrastructure are in place to support all of its missions with over $50 \mathrm{X}$-ray, optical and nuclear diagnostic systems and the ability to shoot cryogenic targets and DT layered capsules. The NIF has also been qualified for the use of tritium and other special materials as well as to perform high-yield experiments and classified experiments. Implosions with record indirect-drive neutron yield of $7.5 \times 10^{14}$ neutrons have been achieved. NIF, a Nd:Glass laser facility, is routinely operating at $1.6 \mathrm{MJ}$ of ultraviolet $(3 \omega)$ light on target with very high reliability. It recently reached its design goal of $1.8 \mathrm{MJ}$ and $500 \mathrm{TW}$ of $3 \omega$ light on target, and has performed target experiments with 1.9 MJ at peak powers of $410 \mathrm{TW}$. The National Ignition Campaign (NIC), an international effort with the goal of demonstrating thermonuclear burn in the laboratory, has been making steady progress toward achieving ignition. Other experiments have been completed in support of high-energy science, materials equation of state, and materials strength. In all cases, records of extreme temperatures and pressures, highest neutron yield and highest energy densities have been achieved. This paper will describe the unprecedented experimental capabilities of the NIF and the results achieved so far on the path toward ignition.
\end{abstract}

\section{Introduction}

The National Ignition Facility (NIF), located at Lawrence Livermore National Laboratory (LLNL) in Livermore, California, is the world's largest and most energetic laser facility for inertial confinement fusion (ICF) research. NIF is the first laser system designed to demonstrate ignition and thermonuclear burn of deuterium-tritium-filled ICF capsules [1]. High-power, high-energy lasers such as NIF, the OMEGA laser at the University of Rochester, and other facilities around the world can compress and heat material, producing unique states of matter and unique radiation environments in the laboratory. These conditions are of interest for High Energy Density Science (HEDS) supporting the NIF missions in national security and fundamental science [2]. Achieving ignition on NIF will not only support the national security mission, but will demonstrate the target physics basis of inertial fusion for energy production. The NIF has been operational and conducting experiments since late in 2009 . The combination of laser, target, and diagnostic capabilities available at NIF make it an unprecedented instrument for the advancement of ICF and other areas of HEDS. A primary goal of the National Ignition Campaign (NIC) on the NIF is to demonstrate fusion ignition and burn via ICF-a goal that has long been recognized as a physics and technology grand challenge. The NIC is rapidly moving forward with new experimental results and code development, developing a large body of knowledge. The NIC approach to ignition utilizes indirect drive with a cryogenic-filled capsule inside of a high- $Z$ hohlraum [3]. Experiments are ongoing to study the four major control variables for ignition: symmetry, fuel adiabat, shell velocity, and mix. Experiments have demonstrated that the techniques developed can observe and control the effects of these variables. Cryogenic layering of the DT fuel with the DT-layer quality required for ignition has been demonstrated, and symmetric implosions at hohlraum temperatures of $300 \mathrm{eV}$ have been achieved in 1.3- to 1.7-MJ experiments with good absorption and low fast-electron preheat. Final fuel $\rho$ r (areal density) is now at about $85 \%$ of the ignition point design and implosion velocities are about $90 \%$ of that required for ignition.

\section{National Ignition Facility}


The NIF is a 192-beam Nd-glass laser for ignition experiments. The facility, shown in Figure 1, consists of two laser bays, a control room and the target area as well as two switchyards and four capacitor bays. NIF
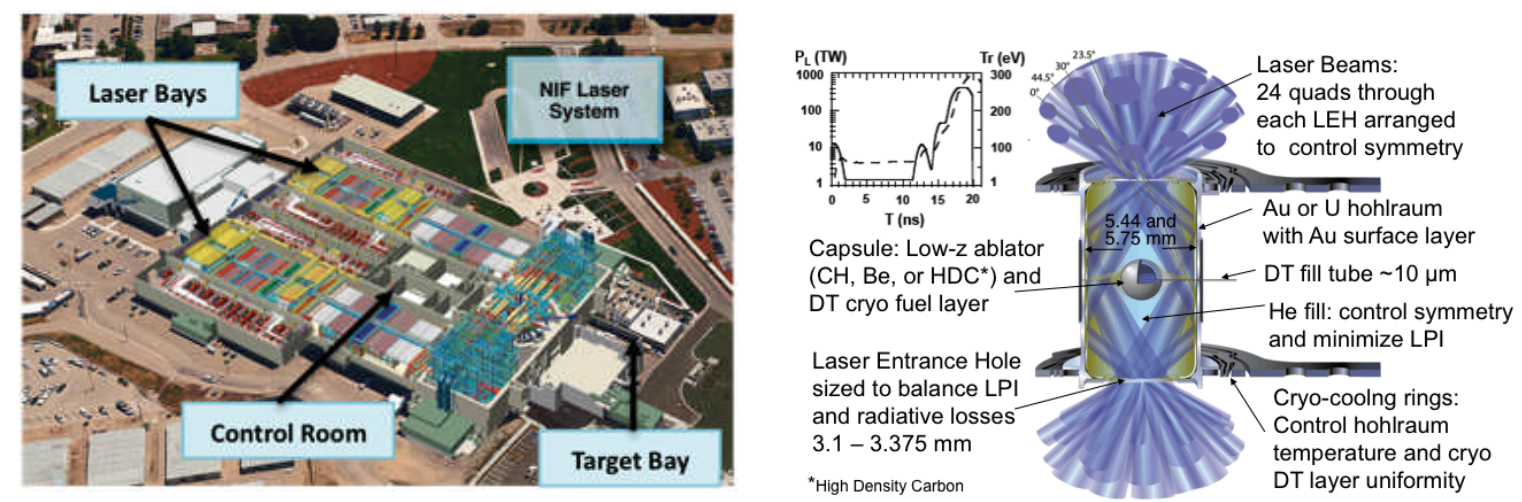

has been operational since the fall of 2009, focusing primarily on physics experiments in support of its missions. NIF has performed experiments for HEDS, x-ray effects, and other national security missions, and for fundamental science. It has executed over 300 target experiments that have resulted in a number of peer-reviewed publications.

FIG. 1. On the left, the NIF facility with the major areas of the facility designated and a cutaway showing the beam path. The right figure shows the NIC indirect-drive ignition point design.

The laser has reached its design goal [4] of delivering 1.8 MJ of frequency-converted $0.35-\mu \mathrm{m}$ light to a target at a peak power of $500 \mathrm{TW}$. The beams irradiate the target in two cones from top and bottom for indirect-drive implosions of ICF capsules. In July 2012, ignition physics experiments were conducted using 1.9 MJ and 420 TW of laser energy/power on target.

Over 50 optical, x-ray, gamma, neutron and charged particle diagnostics are available at NIF, with additional advanced diagnostics planned. The NIF diagnostic team includes individuals from the academia, industry, and national laboratories in the US and several international collaborations.

During the past year, additional hardware has been added to manage the unique conditions produced by ignition experiments. Target experiments sometimes contain beryllium and uranium. Equipment and procedures have been developed to safely handle these hazardous materials. Tritium processing and handling equipment have also been installed to manage the tritium from ignition targets. An ignition shot at full yield of $20 \mathrm{MJ}$ of fusion energy produces nearly $10^{19}$ neutrons. Shielding and monitors have been installed to ensure safe operations. The facility is now qualified for full-yield ignition experiments.

\section{National Ignition Campaign (NIC) and Progress Towards Ignition}

The NIC was formed in 2005 as a comprehensive program to provide the capabilities for performing ignition experiments, to develop the physics basis for ignition, and to conduct the initial ignition experimental campaign. The goal of NIC is to demonstrate a reliable and repeatable ignition platform and to develop NIF as a user facility for its multiple missions. The NIC is a partnership that includes General Atomics, LLNL, Los Alamos and Sandia National Laboratories, the University of Rochester's Laboratory for Laser Energetics, and a number of other collaborators including Lawrence Berkeley National Laboratory, the Massachusetts Institute of Technology, the U.K. Atomic Weapons Establishment, and the French Atomic Energy Commission [5].

In the indirect-drive configuration being used in the current ignition campaign, the capsule is placed inside a cylindrical cavity of a high- $Z$ metal (a hohlraum), and the implosion pressure is provided by focusing 
laser energy onto the interior walls of the hohlraum and converting it to X-rays (see Fig 1). In central hot spot ignition (HSI), the small (few \% of the total DT fuel mass), high-temperature central part of the imploded fuel provides the "spark," which ignites the cold, high-density portion of the fuel. The NIC goal is to achieve ignition and gain equal to or greater than 1 (fusion yield/laser input energy) with HSI targets. The NIF ignition experiments use a centimeter-scale $\mathrm{Au} / \mathrm{U}$ hohlraum containing a 2-mm-diameter, thinwalled plastic, beryllium or high-density carbon capsule filled with a mixture of deuterium and tritium. NIF scientists have demonstrated that NIF can meet the ignition energy performance goals of $1.8 \mathrm{MJ}$ simultaneously with the requirements for temporal pulse shaping, focal-spot conditioning, and peak power of $500 \mathrm{TW}$ for representative ignition pulses.

The NIC is proceeding in phases. In the first "drive" phase, the cryogenic hohlraums utilizing capsules without DT layers demonstrated that NIF could produce the necessary radiation drive and radiation flux uniformity on the capsule as a function of time. In the second "capsule" optimization phase, a variety of experimental platforms were, and are, being used to optimize hohlraum symmetry, shock timing, velocity and mass ablated to move systematically toward the conditions in the imploding capsule required for ignition. In the third phase, capsules with cyro-layered fuel are then used to evaluate the improvement in imploded fuel conditions expected from capsule optimization experiments.

The drive experiments have measured drive temperatures of $300 \mathrm{eV}$ with laser absorption of $80-85 \%$ at laser energies up to 1.9 MJ and radiation symmetry control sufficient for ignition [6]. Energy transfer between crossing laser beams is an important effect and can be controlled and used to modify the hohlraum environment. New models have been developed for the radiating plasma and are being used to refine the ignition target design $[7,8,9,10,11,12]$. The fourth phase, or DT ignition campaign, began in September 2011, and implosions with cryolayers have been conducted with laser energies up to $1.7 \mathrm{MJ}$ and peak power close to $500 \mathrm{TW}$. Hot spot ion temperatures of $3.7 \mathrm{keV}$, main fuel density of 850 of $\mathrm{g} / \mathrm{cm}^{3}$, main fuel areal density ( $\rho r$ ) of $\sim 1.3 \mathrm{~g} / \mathrm{cm}^{2}$ and neutron yields of $6-8 \times 10^{14}$ have been achieved.

One metric for expressing progress toward ignition is the Experimental Ignition Threshold Factor (ITFX). The ITFX depends upon the experimentally measured neutron yield and the ratio of down-scattered neutrons to un-scattered neutrons (dsr) and is proportional to areal density $\rho r$ of the fuel (the DT fuel $\rho r$ $\sim 20 \times \mathrm{dsr}$ in $\mathrm{g} / \mathrm{cm}^{2}$ ). ITFX is related to the generalized Lawson criterion and is normalized so that an ITFX of 1 is defined as the condition in which the chance of a yield greater than $1 \mathrm{MJ}$ on a given shot is $50 \%$. Progress toward ignition can be graphically represented as shown in Figure 2 in which cryolayered target neutron yield is plotted versus the dsr where the contours drawn represent constant ITFX.
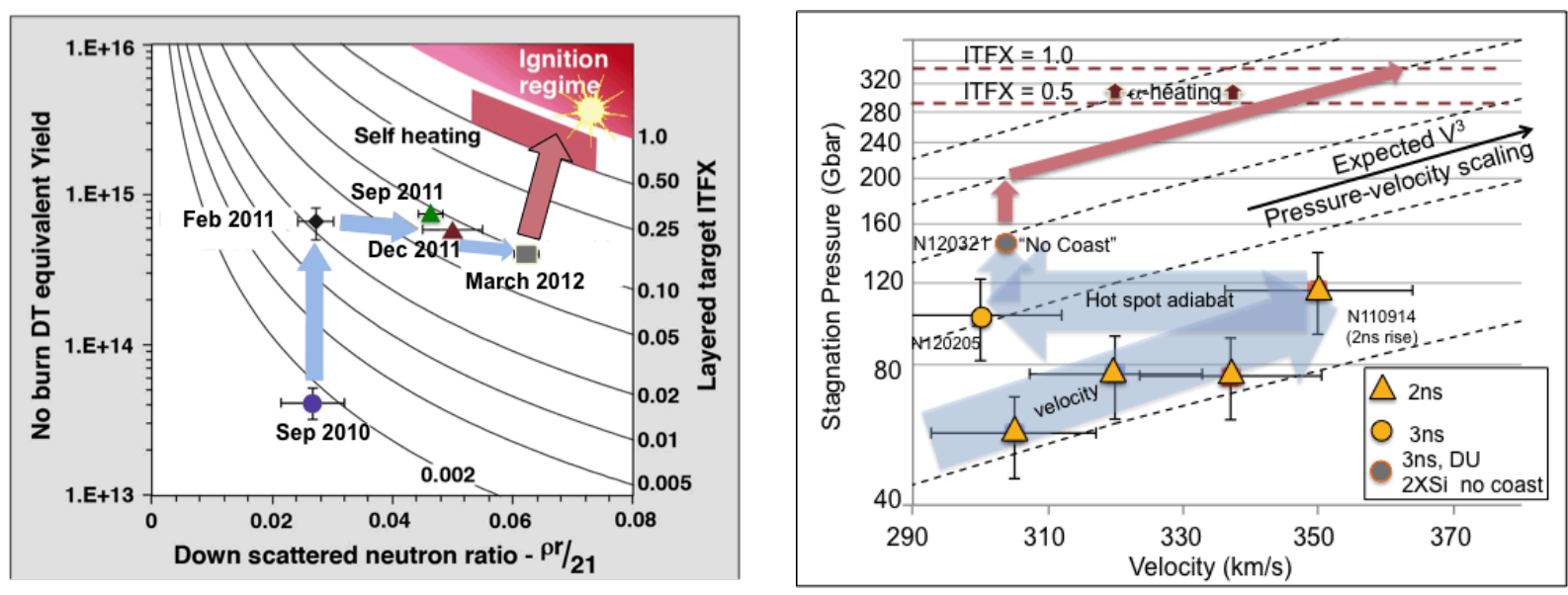

FIG. 2a:The fuel $\rho r$ is at about 85\% of the ignition point design and "no burn" yields are $20 \%$ of that required for the alpha-dominated self-heated regime. FIG. $2 \boldsymbol{b}$ shows the stagnation pressure as a function 
of peak implosion velocity for a variety of targets and implosion parameters. In both figures the blue arrows show progress made while maroon arrows indicate projected improvements with thicker ablators driven with 1.8-2 MJ and $500 \mathrm{TW}$.

The yields plotted are those arising from compression during the implosion process and do not include enhancements from alpha deposition that become significant as the implosion quality approaches that required for ignition. This yield is adopted because it provides a metric for the underlying quality of the implosion needed to get into a regime where the alpha particle deposition becomes significant. The ignition regime is above ITFX of 1 , while the alpha-dominated regime, in which the yield enhancement from alpha particle deposition exceeds the yield from compression alone, occurs for an ITFX of $\sim 0.3-0.5$. The progress in ITFX shown in Figure 2 can be summarized as follows:

- Improvement in ITFX between the first cryogenic layered implosion in September 2010 and February 2011, mostly in the neutron yield, was achieved by increasing the laser power and performing shock-tuning experiments.

- During 2011, ITFX was increased to $\sim 0.08$. This was achieved with improved shock timing as well as by increasing implosion velocity using Si-doped rather than Ge-doped $\mathrm{CH}$ ablators, increasing laser performance, and improving implosion symmetry.

- In 2012, the slope of the rise of the fourth pulse was decreased, producing an implosion with higher compression and lower adiabat. The length of the fourth pulse was extended to better maintain compression of the shell during final convergence, producing a higher dsr, or $\rho$, resulting in an improved ITFX of $\sim 0.1$, although at a lower velocities and decreased drive energy.

Although ITFX only increased by about 25\% between September 2011 and March 2012, the recent implosions provide a much better path to ignition. As shown in Figure 2, recent experiments have not only produced areal densities of $\sim 85 \%$ of the ignition point design, but the pressure in the hot spot has increased to $\sim 140 \mathrm{Gbars}, \sim 40 \%$ of the ignition point design. These conditions were obtained using peak powers of $320 \mathrm{TW}$ with implosion velocity of $\sim 300 \mathrm{~km} / \mathrm{s}$. The stagnation pressure was first increased by increasing the peak power (and hence increasing the implosion velocity) with the slope of the fourth pulse kept at 2 ns. The peak power was then reduced to $320 \mathrm{TW}$ and the slope was increased to $3 \mathrm{~ns}$ maintaining the higher pressure while driving the fuel compression on a lower adiabat. While staying at a peak power of $320 \mathrm{TW}$, the peak stagnation pressure was then increased to 140 Gbars by extending the peak power pulse to maintain compression prior to final stagnation (indicated by "no coast" on Figure 2). By operating with thicker ablators and peak power up to $500 \mathrm{TW}$, increases are expected in hot-spot pressures to between 320-350 Gbars and in implosion velocities to the $370 \mathrm{~km} / \mathrm{s}$ required for ignition.

Recent progress in ignition has been achieved by a combination of expanding the physics issues considered in experiments and enhancing facility experimental capability. Experimental platforms have been developed for optimizing key implosion attributes including shock timing in the cryogenic fuel, radiation symmetry at early time in the initial picket of the pulse, symmetry of the imploded capsule at peak compression, and radiography of the imploding shell to enable measuring implosion velocity and mass ablated from the imploding shell.

As target performance has improved, the effect of mix on implosion performance has been observed, quantified, and is being investigated. In addition, the experimental platforms have become more capable, and steady advances in laser performance enabled the longer pulses that provided the increase in laser energy used in recent experiments. During March and April 2012, the NIF laser routinely delivered pulse energies of 1.45-1.9 MJ with powers over $400 \mathrm{TW}$, and NIF has also performed target experiments at the full design energy and power of $1.8 \mathrm{MJ}$ and $500 \mathrm{TW}$. The planned experimental campaign will be exploring the performance of ablator shells that are 10-20\% thicker than current targets, driven by laser power and energy up to $500 \mathrm{TW}$ and $1.8 \mathrm{MJ}$, respectively. It is expected these experiments will allow an 
increase in the implosion velocity achievable without significant mix.

\section{NIF as an experimental platform for fundamental science}

The importance of NIF for fundamental science has been discussed in a number of reports [13, 14], including most recently in the report of a workshop jointly sponsored by the NNSA and the Department of Energy Office of Science [15]. The NIF laser's unprecedented power, precision, and reproducibility, coupled with sophisticated target fabrication and diagnostic capabilities put in place via the NIC and other experimental campaigns, is enabling leading edge experiments in the study of matter at extreme conditions.

As an example, in the past year, scientists from the University of California (Berkeley), Princeton University, LLNL, and elsewhere, building on experience at OMEGA and other facilities, conducted four experiments on NIF that compressed diamond to peak pressures exceeding 50 MBars - conditions never previously encountered in the laboratory and similar to those found in planetary interiors. An international collaboration involving scientists from LLNL, LANL, LBNL, and other institutions has also observed on NIF the first low-energy (sub-MeV) neutrons produced in an ICF implosion. This will allow studies of nuclear excited states and other effects of importance to nucleosynthesis. More generally, this experiment represents the first step in an effort to realize the unique opportunity for nuclear physics afforded by the dense, hot, high-neutron-flux environments produced in NIF ICF implosions. Other astrophysics-related experiments conducted at NIF to date include initial experiments in supernova hydrodynamics performed by a team of researchers including scientists from the University of Michigan, Florida State University, and LLNL. Other experiments proposed include an effort to produce the first relativistic electron-positron pair plasmas in the laboratory, enabling experiments on a state of matter found in gamma-ray bursts, black holes and active galaxies.

NIF scientists have made progress towards installation of the infrastructure required to support these and other experiments planned by the fundamental science research community. In addition to the laser, diagnostic, and other facility capabilities implemented by the NIC, LLNL has created a "HEDS Campus" to support visiting researchers. A successful call for proposals for fundamental science at NIF was also executed in 2009-2010. This competition received a strong response, with approximately one-half of the proposals from US academic institutions and one quarter each from institutions outside the US and the NNSA laboratories. The experimental campaigns have begun. A total of 10 teams are now pursuing fundamental science experiments at the NIF. To support the science on NIF a NIF User's Group has been formed and a Workshop with 162 participants from 16 countries was held at LLNL in February 2012.

\section{NIF Experiments in support of High Energy Density Stewardship Science (HEDSS)}

NIF is rapidly emerging as the premier facility in the world for the study of matter at extreme conditions of temperature and pressure. As mentioned above, the NIF laser's unprecedented power, precision, and reproducibility, coupled with sophisticated target fabrication and diagnostic capabilities put in place via the NIC and other experimental campaigns, is enabling leading-edge experiments in support of the Stockpile Stewardship Program (SSP). A key mission driver for the SSP is to deliver predictive, physicsbased capabilities to enable the assessment of the safety, reliability, and performance of the U.S. nuclear stockpile in an era without nuclear testing. During the operation of a thermonuclear weapon, extreme conditions of temperatures, pressures, and densities are produced that do not occur anywhere else except in the interior of stars and planets. 
High Energy Density Stewardship Science (HEDSS) experiments on NIF study matter in these extreme conditions to understand the physics when matter is heated to high temperature and density. An example of a platform for radiation transport experiments is shown in Figure 3a. Hohlraums are used to produce a thermal radiation source that irradiates complex structures in a high- $Z$ foam. The time dependence of the radiation transport is studied through these complex structures. Figure $3 \mathrm{~b}$ shows the platform for equation of state (EOS) measurements. By carefully tailoring the time-dependence of the laser power entering the hohlraum, the x-ray power incident on the ablator gradually increases, with a gradually increasing pressure in the EOS sample. This method provides very high quasi-isentropic conditions inside planar EOS samples.

A number of experimental platforms are being developed that can reach the extreme temperature and density regimes previously inaccessible in the laboratory. NIF has already completed several HEDSS campaigns in support of SSP in radiation transport, complex hydrodynamics, and materials dynamics. Over the past two years, several different experimental campaigns totalling more than 60 shots in support of HEDSS have studied different aspects of high energy density physics.
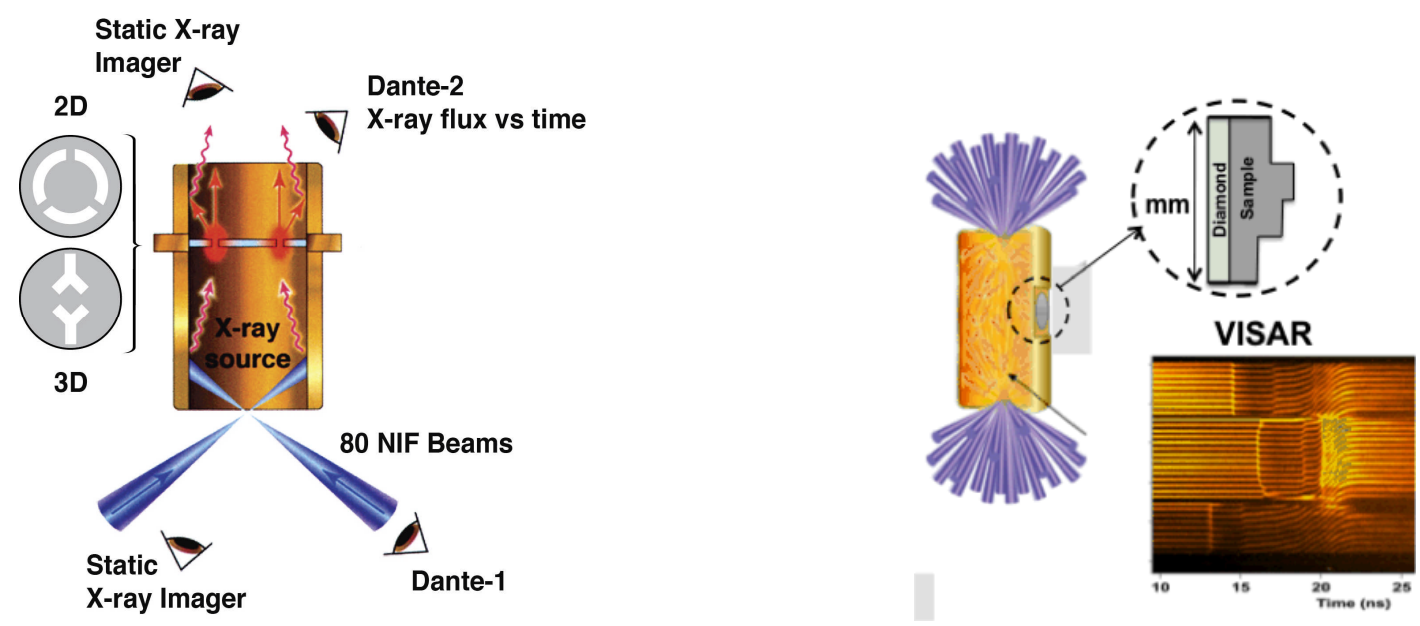

FIG. 3a. Experimental configuration on NIF for radiation transport experiments. FIG. $3 \boldsymbol{b}$. Experimental configuration and Velocity Interferometer System for Any Reflector (VISAR) data for EOS experiments on the NIF.

\section{Summary}

A primary goal of the National Ignition Campaign (NIC) is to demonstrate fusion ignition and burn by inertial confinement fusion (ICF) - a goal that has long been recognized as a physics and technology grand challenge. The NIC is rapidly moving forward with new experimental results and code development, generating a large body of knowledge. Continuing progress in all aspects of ICF technology, operational systems, experimental platforms and codes has enabled a robust experimental program to understand the physics of ignition-scale implosions and to iteratively improve implosion performance as the knowledge base has grown. The NIF laser's unprecedented power, precision, and reproducibility, coupled with sophisticated target fabrication and diagnostic capabilities put in place via the NIC and other experimental campaigns, is enabling leading-edge experiments in support of other mission areas including Stockpile Stewardship Program (SSP) and fundamental science.

\section{Acknowledgements}


This work performed under the auspices of the U.S. Department of Energy by Lawrence Livermore National Laboratory under Contract DE-AC52-07NA27344.

\section{References}

[1] G.H. Miller, E.I. Moses, C.R. Wuest, "The National Ignition Facility: enabling fusion ignition for the 21st century," Nucl. Fusion, 44 S228 (2004).

[2] R. Davidson et al., "Frontiers in High Energy Density Physics: The X-Games of Contemporary Science," The National Academies, Washington, DC (2003).

[3] J.D. Lindl, "Inertial confinement fusion: the quest for ignition and energy gain using indirect drive," American Institute of Physics Press, New York (1998).

[4] G.A. Haynam, P.J. Wegner, J.M. Auerbach et al., "National Ignition Facility laser performance status," Appl. Opt. 46, 3276 (2007).

[5] "National Ignition Campaign Execution Plan,” LLNL report UCRL-AR-213718, NIF-0111975-AA (2005)

[6] S.H. Glenzer et al., "Demonstration of Ignition Radiation Temperatures in Indirect-Drive Inertial Confinement Fusion Hohlraums," Phys. Rev. Lett. 106, 085004 (2011).

[7] N.B. Meezan, L.J. Atherton, D.A. Callahan et al., "National Ignition Campaign Hohlraum Energetics," Phys. Plasmas 17, 056304 (2010).

[8] S.H. Glenzer et al., "Symmetric Inertial Confinement Fusion Implosions at Ultra-High Laser Energies," Science 327, 1228 (2010).

[9] P. Michel, L. Divol, E.A. Williams et al., "Tuning the Implosion Symmetry of ICF Targets via Controlled Crossed-Beam Energy Transfer," Phys. Rev. Letters 102, 025004 (2009).

[10] G.A. Kyrala, J.L. Kline, S. Dixit, S. Glenzer et al., "Symmetry tuning for ignition capsules via the symcap technique," Phys. Plasmas 18, 056307 (2011).

[11] R.P.J. Town, M.D. Rosen, P.A. Michel et al., "Analysis of the National Ignition Facility ignition hohlraum energetics experiments," Phys. Plasmas 18, 056302 (2011).

[12] M.D. Rosen, H.A. Scott, D.E. Hinkel, E.A. Williams, D.A. Callahan, R.P. J. Town, L. Divol, P.A. Michel, W.L. Kruer, L.J. Suter, R.A. London, J.A. Harte, and G.B. Zimmerman, "The role of a Detailed Configuration Accounting (DCA) atomic physics package in explaining the energy balance in ignition scale hohlraums," High Energy Density Physics 7, (3), 180 (2011).

[13] E.I. Moses, R.N. Boyd, B.A. Remington, C.J. Keane, and R. Al-Ayat, "The National Ignition Facility: Ushering in a New Age for High Energy Density Science," Physics of Plasmas 16, 41006 (2009).

[14] National Research Council, High Energy Density Physics: The X-Games of Contemporary Science, National Academies Press, Washington, DC, 2003.

[15] U.S. Department of Energy National Nuclear Security Administration (NNSA) and Office of Science, Basic Research Directions for Science at the National Ignition Facility, U.S. Department of Energy, Washington DC, 2011. 